\section{Commentary: Superior cavopulmonary connection: A false sense of security?}

\author{
Tain-Yen Hsia, MD
}

When Marc de Leval introduced the total cavopulmonary connection in 1988, it was, like all other previous versions of the Fontan operation, meant to be performed as a single-stage procedure. The initial concept of preceding the Fontan with an interval superior cavopulmonary connection (SCPC) was to reduce the mortality and morbidity in high-risk Fontan patients. However, as a staged approach became popular for management of hypoplastic left heart syndrome (HLHS), it is now the standard practice for most patients with single-ventricle hearts, regardless of anatomy. ${ }^{1}$ In addition to early volume unloading the single right ventricle and minimizing the acute change in ventricular mass to volume ratio seen with single-stage Fontan, SCPC confers an ever-growing source of pulmonary blood flow that avoids the flaws associated with systemic-topulmonary artery shunts or bilateral pulmonary arterial bands. In fact, SCPC is so preferable to stage- 1 circulation for HLHS palliation that some advocate transition to stage2 operation before a patient reaches age 3 months. ${ }^{2}$ SCPC renders such a well-tolerated circulation that the assumption is our patients are out of the danger from interstage attrition. Therefore, for a long time, we have focused our attention on mitigating attrition between stages 1 and 2 , as well as the long-term sequelae of the Fontan state. Getting an HLHS patient to SCPC is cause for celebration.

As it turns out, our sense of security regarding the SCPC is false. Building upon a growing number of other smaller studies, including 1 derived from the Single Ventricle Reconstruction Trial, the exceptional group at Children's Hospital of Philadelphia further raises awareness about

\footnotetext{
From the Department of Pediatric Cardiac Surgery, The Heart Center at Arnold Palmer Hospital for Children, Orlando, Fla.

Disclosures: The author reported no conflicts of interest.

The Journal policy requires editors and reviewers to disclose conflicts of interest and to decline handling or reviewing manuscripts for which they may have a conflict of interest. The editors and reviewers of this article have no conflicts of interest.

Received for publication Dec 3, 2020; revisions received Dec 3, 2020; accepted for publication Dec 3, 2020; available ahead of print Dec 17, 2020.

Address for reprints: Tain-Yen Hsia, MD, Department of Pediatric Cardiac Surgery, Arnold Palmer Hospital for Children, 92 W Miller St, MP307, Orlando, FL 32806 (E-mail: tain-yen.hsia@orlandohealth.com).

J Thorac Cardiovasc Surg 2021;162:396-7

$0022-5223 / \$ 36.00$

Copyright (c) 2020 by The American Association for Thoracic Surgery

https://doi.org/10.1016/j.jtcvs.2020.12.035
}

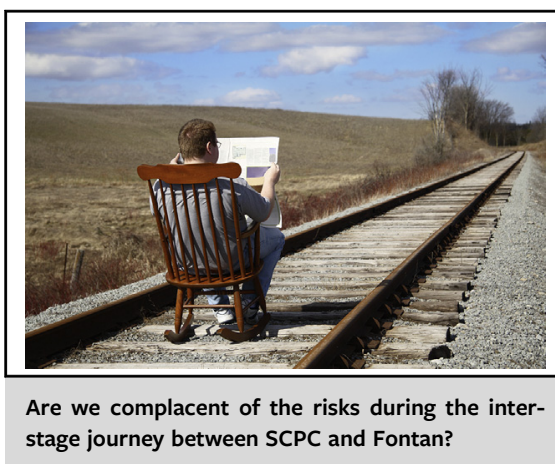

CENTRAL MESSAGE

The interstage attrition between superior cavopulmonary connection to Fontan remains significant for patients with hypoplastic left heart syndrome.

the unrelenting attrition of HLHS patients between SCPC and Fontan. ${ }^{3}$ The authors tapped into their massive institutional HLHS experience over 3 decades to reveal that interstage attrition (ie, death or heart transplantation) between stages 2 and 3, in an 851-patient cohort, is significant and has not changed at all. In addition to confirming an $8 \%$ $10 \%$ rate of attrition, new insights uncovered in this study include identifying the use of right ventricle-to-pulmonary artery (RV-PA) shunt at stage 1 as an important antagonist. Other risk factors, such as needing atrioventricular valve repair and a longer post-SCPC course, were reaffirmed to influence interstage outcomes. But not everything is doom and gloom. The good news is that many of the significant hazards that contribute to poor stage 1 outcomes, such as low gestational age, genetic anomalies, anomalous pulmonary venous drainage, and intact atrial septum, are no longer pertinent. This survival effect suggests that, for HLHS patients with these risk factors, achieving SCPC circulation is a major milestone that renders those nonmodifiable or anatomic features insignificant.

Nonetheless, as a descriptive study based on historical information and incomplete details, there are areas of missed opportunities that raise more questions. One of these is on the reported doubling rate of attrition within the 2 later eras in patients who received RV-PA shunts at stage 1, compared with those who had modified Blalock-Taussig shunts. Because the inter 2-to-3 stage attrition remained the same throughout the 4 eras, are the authors suggesting that had they stayed with a modified Blalock-Taussig shunt 
in all patients, the later era outcomes would have been better? It is also unfortunate that the authors were unable to provide insights into whether ventricular dysfunction or poor pulmonary arterial growth (or both) was the mechanistic driver for the worse attrition seen in RV-PA shunt patients. Similarly, the need for atrioventricular valve repair is only a surrogate for significant regurgitation. Without complete echocardiographic information before and after the repair, we are left wanting more. Finally, although the authors tell us that the mode of failure for those who underwent heart transplantation was primarily ventricular dysfunction, they were unable to account for the cause of death in the 52 who died. Were they not candidates for transplantation because of poor pulmonary function or pulmonary hypertension?

It is quite right that the authors used this study as a call to arms to redouble efforts to reduce the attrition rate between SCPC and Fontan, but rather than admitting failure, I would play devil's advocate here. The lack of improvement of the interstage attrition over the 3 decades and 4 eras, rather than due to our lack of understanding or diligence, may very well be due to the nature of the cavopulmonary circulation. Being a transitional circulatory arrangement that typically lasts 2 to 3 years, SCPC promotes venous return and pulmonary blood flow without a ventricular power source. Perhaps, just like the Fontan circulation, despite all our efforts to optimize its construction and understanding of its long-term effects, it is a fundamentally flawed physiology with a continuing and unyielding influence on quality of life and failure. We should continue to invest in interstage management and monitoring, yet we need to entertain the possibility that despite our best industry and dedication there will always be significant number of single-ventricle patients not reaching Fontan.

Finally, at its inception in the 1940s and 1950s, cavopulmonary anastomosis neither pertained to the Fontan circulation nor applied to single-ventricle pathologies. Rather, cavopulmonary connection described the various procedures to connect the SVC to the right pulmonary artery to treat a range of defects where there is insufficient pulmonary blood flow, such as tetralogy of Fallot. Later, the term bidirectional was added to denote modifications where the superior venous return is connected to both the right and left pulmonary arteries. Therefore, the authors should be commended for adopting SCPC to describe stage 2 circulation, rather than the often used bidirectional cavopulmonary connection. After all, Fontan in all its forms (including the total cavopulmonary connection) also provides pulmonary blood flow to both sides.

\section{References}

1. Mazzera E, Corno A, Picardo S, Di Donato R, Marino B, Costa D, et al. Bidirectional cavopulmonary shunts: clinical applications as staged or definitive palliation. Ann Thorac Surg. 1989;47:415-20.

2. Viegas M, Diaz-Castrillon CE, Castro-Medina M, Da Fonseca Da Silva L, Morell VO. Bidirectional Glenn procedure in patients less than 3 months of age: a 14-year experience. Ann Thorac Surg. 2020;110:622-9.

3. Lawrence KM, Ittenbach RF, Hunt ML, Kaplinski M, Ravishankar C, Rychik J, et al. Attrition between the superior cavopulmonary connection and the Fontan procedure in hypoplastic left heart syndrome. J Thorac Cardiovasc Surg. 2021;162:385-93. 